\title{
A efetividade das intervenções educativas em saúde bucal: revisão de literatura
}

\author{
The effectiveness of educational interventions in oral \\ health: a literature review
}

\author{
Isabel Lemkuhl' ${ }^{1}$ Maria Vitória Cordeiro de Souzaํㅜ, Andreia Morales Cascaes², \\ João Luiz Bastos ${ }^{1}$
}

\begin{abstract}
Resumo
Visando diminuir impactos negativos dos agravos bucais, intervenções educativas em saúde bucal têm sido propostas e avaliadas. Entretanto, a efetividade das iniciativas publicadas a partir de 2004 ainda não constituiu objeto de escrutínio acadêmico. Este trabalho objetivou revisar as intervenções educativas e estimar seu impacto sobre as condições de saúde bucal. Consultou-se a base de dados MEDLINE via PubMed, com termos livres e controlados, para identificar intervenções educativas publicadas no período de 2003 a 2013. Analisaram-se e sintetizaram-se qualitativamente os resultados dos estudos originais. Nos 37 estudos revisados, observou-se que as intervenções produziram resultados clínicos inconsistentes em curto prazo, incluindo a redução de placa bacteriana (melhor resultado [MR] -88,9\%; pior resultado [PR] $+29,7 \%$ ), sangramento gengival (MR $-77,8 \%$; PR $+72,2 \%$ ) e menor incremento de cárie (MR -39,5\%; PR $+961,1 \%$ ) e de cálculo dentário $(+16,2 \%)$. Por sua vez, houve maior redução dos desfechos avaliados, quando os participantes receberam acompanhamento clínico intensivo, sugerindo que este é um aspecto importante das intervenções. De modo semelhante às revisões prévias da literatura, observou-se que as intervenções educativas para prevenção, controle ou redução de agravos bucais não apresentaram efeitos consistentes.
\end{abstract}

Palavras-chave: educação em saúde; promoção da saúde; saúde bucal; revisão.

\begin{abstract}
Educational interventions have been proposed and evaluated in order to reduce the negative impacts of oral health outcomes. However, the effectiveness of the initiatives published from 2004 has not been scientifically scrutinized yet. This study aimed to review the literature on educational interventions and estimate their impacts on oral health outcomes. A literature review, using a search query with free and controlled terms, was carried out in Medline via PubMed, to identify educational interventions published between 2003-2013. The original results were analyzed and qualitatively summarized. In the 37 studies reviewed, it was observed that educational interventions tended to produce inconsistent short term clinical results, including dental plaque reduction (best result $[\mathrm{BR}]-88.9 \%$; worst result $[\mathrm{WR}]+29.7 \%)$, reduced gingival bleeding (BR $-77.8 \%$; WR $+72.2 \%$ ), reduced dental caries (BR $-39.5 \%$; WR $+961.1 \%)$ and reduced dental calculus (+16.2\%). In turn, there was a greater reduction in the outcomes evaluated, when participants received intensive clinical monitoring, suggesting that this is an important aspect of the interventions. Similarly to previous literature reviews, this study showed that educational interventions aimed at preventing, controlling or reducing oral diseases are not consistent.
\end{abstract}

Keywords: health education; health promotion; oral health; review.

${ }^{1}$ Dentista. Doutor em Epidemiologia do Departamento de Saúde Pública da Universidade Federal de Santa Catarina (UFSC) - Florianópolis (SC), Brasil. ${ }^{2}$ Dentista. Doutora em Epidemiologia do Departamento de Odontologia Social e Preventiva da Faculdade de Odontologia da Universidade Federal de Pelotas (UFPEL) - Pelotas (RS), Brasil.

Trabalho realizado na Universidade Federal de Santa Catarina (UFSC) - Florianópolis (SC), Brasil.

Endereço para correspondência: João Luiz Bastos - Rua Roberto Sampaio Gonzaga, s/n - Trindade - CEP: 88040-970 - Florianópolis (SC), Brasil - Email: joao.luiz.epi@gmail.com

Fonte de financiamento: nenhuma.

Conflito de interesses: nada a declarar. 


\section{INTRODUÇÃO}

A cárie e a doença periodontal são os agravos bucais mais prevalentes no mundo e no Brasil. Cerca de 60,0 a 90,0\% da população mundial é acometida por cárie ${ }^{1}$. Por sua vez, a doença periodontal severa acomete de 15,0 a 20,0\% dos adultos entre 35 e 44 anos de idade ${ }^{1}$. No Brasil, dados da última Pesquisa Nacional de Saúde Bucal (SBBrasil 2010) revelam que a cárie atinge aproximadamente $76,0 \%$ dos adolescentes de 15 a 19 anos e quase a totalidade dos adultos e dos idosos ${ }^{2}$. Bolsas periodontais foram encontradas em 19,0\% dos adultos brasileiros de 35 a 44 anos $^{2}$.

Esses agravos acarretam múltiplos impactos negativos e sofrimento, incluindo a perda de dentes, dor, baixa autoestima e halitose ${ }^{3,4}$. Além de afetarem a qualidade de vida, demandam elevados custos para os serviços de saúde e, de forma mais ampla, para a sociedade. Tais problemas bucais estão distribuídos de forma desigual na população e são especialmente prevalentes em grupos sociais mais vulneráveis, incluindo populações sem acesso à água tratada e com alto consumo de alimentos açucarados ${ }^{1}$.

Tendo em vista que essas condições bucais - a doença periodontal e a cárie - são passíveis de prevenção por meio de escovação dentária diária, do contato regular com fontes de fluoretos ${ }^{5,6}$ e do controle do consumo de açúcar ${ }^{7}$, intervenções educativas que enfatizam alteração desses comportamentos têm sido propostas na literatura. Tais estratégias visam diminuir tanto a ocorrência e o impacto que os agravos bucais acarretam para os indivíduos quanto as desigualdades sociais a eles relacionadas. Particularmente, uma revisão de iniciativas publicadas entre 1982 e 1994 demonstrou que, de modo geral, essas intervenções são associadas à redução de placa e de sangramento gengival, porém sem significância do ponto de vista clínico e apenas em curto prazo ${ }^{8}$.

Em geral, esta revisão, bem como outros estudos ${ }^{8-10}$, concluiu que informações sobre saúde bucal transmitidas em diversas intervenções educativas são importantes, mas insuficientes para promover mudanças nos comportamentos relacionados à saúde bucal, com a consequente melhoria das condições bucais. A qualidade metodológica dos estudos tem sido considerada insuficiente, e a maioria das intervenções utilizou abordagens educativas tradicionais, pautadas na simples transmissão de conhecimentos, sem enfrentar complexidade dos determinantes sociais dos comportamentos e do processo saúde-doença.

Resultados similares foram encontrados em uma outra revisão de literatura sobre o tema, publicada no ano de $2005^{10}$. No entanto, a efetividade das intervenções educativas, especificamente a partir de 2004, ainda não constituiu objeto de discussão por pesquisadores da área. Embora Satur et al. ${ }^{9}$ tenham recentemente divulgado uma revisão sobre o assunto, abordando estudos publicados até 2006, este trabalho não apreciou exclusivamente estudos de intervenção e consistiu, mais especificamente, de um artigo de opinião. Ademais, as revisões mencionadas avaliaram o resultado das intervenções educativas sobre agravos bucais específicos, principalmente cárie e doença periodontal. O presente estudo, apesar de não se propor a realizar uma metanálise a partir de uma revisão sistemática, objetiva fazer uma análise ampla dos estudos, quantificando alguns de seus elementos para uma apreciação do tema.

Dessa forma, as seguintes perguntas de pesquisa foram consideradas pertinentes no presente trabalho: (1) As intervenções educativas realizadas a partir de 2004 são efetivas, quando comparadas com aquelas publicadas previamente? (2) Qual é o efeito dessas intervenções sobre múltiplos desfechos de saúde bucal? O presente estudo revisou a literatura mais atual sobre o tema, analisando o impacto das intervenções educativas em saúde bucal, sem restrição a agravos específicos.

\section{MÉTODOS}

A revisão da literatura foi realizada por meio de consulta eletrônica à base de dados MEDLINE via PubMed. Foi confeccionada uma estratégia de busca com termos livres e controlados, dividida em dois componentes: o primeiro reuniu os termos relacionados à exposição, ou seja, à intervenção propriamente dita: ("health education, dental" [mesh] OR "oral health promotion" [tiab]); o segundo, termos definidores dos desfechos finais dos estudos: ("oral health" [mesh] OR "attitude to health" [mesh] OR "health knowledge, attitudes, practice" [mesh] OR "oral health behavior" [tiab] OR "oral hygiene" [tiab] OR "oral health status" [tiab]). Os dois grupos de termos foram combinados entre si por meio do operador booleano "AND".

Os estudos incluídos deveriam: (a) isolar o efeito da intervenção educativa sobre o desfecho, comparando-a com sua ausência; (b) analisar desfechos finais clínicos, como o nível de placa dental ou o ataque de cárie dentária, não considerando os resultados intermediários, por exemplo, o aumento do nível de conhecimento pelos participantes; (c) estar disponíveis em línguas acessíveis aos autores - português, espanhol ou inglês; (d) ter sido publicados no período de janeiro de 2003 a fevereiro de 2013. Foi incluído o ano de 2003 pelo fato de a revisão mais recente sobre $\mathrm{o}$ assunto ter abrangido estudos divulgados até $\mathrm{o}$ início desse ano ${ }^{10}$. Estudos sem grupo controle também foram incluídos, na medida em que também ofereciam estimativas indiretas - do impacto das intervenções.

As publicações, identificadas em fevereiro de 2013 no MEDLINE, e o conjunto desses resultados foram submetidos à leitura, realizada de maneira independente por três autores desta revisão. Procedeu-se a leitura do título e do resumo de cada publicação identificada, excluindo-se os trabalhos que não atendiam aos critérios de inclusão. Os trabalhos selecionados por cada autor foram comparados quanto à pertinência, e, nos 
casos de divergência, a inclusão/exclusão foi realizada por meio de consenso. Os artigos com relevância duvidosa foram lidos na íntegra para decisão final quanto à elegibilidade. Os autores de estudos que não continham informações suficientes para análise foram contatados via mensagens de correio eletrônico.

Foi confeccionado um formulário para efetuar a extração de dados dos artigos selecionados, no qual foram registrados: ano de publicação, país onde foi realizado o estudo, idioma em que o trabalho foi publicado, quantidade de participantes que iniciou e concluiu o estudo, tempo de duração da intervenção educativa, idade e sexo dos participantes, material utilizado nas intervenções, acompanhamento dos profissionais durante a intervenção, desfecho analisado e avaliação dos resultados em curto e longo prazo.

Após treinamento dos autores quanto ao correto preenchimento da ficha, os dados foram digitados com auxílio do programa EpiData, versão 3.1, incluindo controles automáticos de consistência e de amplitude. Os dados foram analisados com o pacote estatístico Stata, versão 11.2. A descrição bibliográfica dos artigos incluiu o país onde foi realizado o estudo e o ano de publicação (2003 a 2005, 2006 a 2008 e 2009 a 2013). Em seguida, realizou-se a caracterização da população, que abrangeu: vinculação a grupos específicos (por exemplo, escolares), sexo, idade mínima e idade máxima dos participantes. Posteriormente, realizou-se um detalhamento das intervenções educativas realizadas nos estudos, no qual foram incluídos a quantidade de grupos avaliados, o profissional que realizou a intervenção, o material utilizado, a presença de grupo controle, a existência de acompanhamento clínico, o tempo de duração da intervenção em meses e a realização de avaliação em longo prazo. O efeito das intervenções educativas sobre os desfechos finais foi considerado de longo prazo, quando realizado, no mínimo, seis meses após a conclusão da intervenção - esse período foi definido pelas próprias características dos estudos originais analisados. O tempo de duração da intervenção foi dividido nas seguintes categorias: $0-2,3-5,6-10,11-20,21-30 \mathrm{e}$ 31 ou mais meses. Essa classificação se baseou na distribuição da variável nos estudos originais e procurou evitar a formação de categorias esparsas para a descrição dos dados. Trabalhos com múltiplas publicações veiculando os mesmos resultados tiveram uma única entrada no banco de dados.

Por fim, foi realizada a transformação dos resultados dos desfechos analisados, de forma que os diferentes tipos de medidas dos agravos estudados pudessem ser comparados em termos percentuais, sendo eles: placa, sangramento gengival, cálculo, placa na mucosa (indicador utilizado para avaliar a mucosa de pacientes edêntulos), profundidade à sondagem e cárie. Para tanto, foram realizados os seguintes cálculos: resultado final da avaliação clínica (RF) subtraído do resultado inicial da avaliação clínica (RI) dos participantes em cada grupo, dividido pelo resultado inicial, multiplicado por cem: $((\mathrm{RF}-\mathrm{RI}) / \mathrm{RI})^{\star} 100$. No caso, o RI consistiu no primeiro exame clínico do estudo, que avaliou o estado dos participantes antes da intervenção; o RF, por sua vez, consistiu no último exame clínico, que avaliou a condição bucal de interesse na última etapa do estudo. Por exemplo, se o RI de algum grupo foi 50, e o RF, 30, tem-se $((30-50) / 50)^{\star} 100$, podendo-se observar uma redução de $40,0 \%$ no desfecho investigado.

Dessa forma, o impacto das intervenções em cada um dos desfechos citados, considerando-se os estudos incluídos na presente revisão, foi estimado de duas maneiras: (a) levando-se em conta somente os registros clínicos inicial e final dos participantes; (b) incluindo-se todas as informações clínicas disponíveis dos indivíduos investigados, compreendendo aquelas intermediárias ao início e ao término da intervenção, além da inicial e da final.

Após a limpeza do banco de dados, foram construídas tabelas de contingência com frequências absolutas/relativas das características das intervenções empregadas. Por não se tratar de uma revisão sistemática da literatura, a metanálise não é recomendada no caso da presente publicação - seria enviesado o efeito global estimado a partir de uma seleção menos exaustiva de estudos originais. Ademais, em diversas publicações encontradas neste estudo faltam informações, por exemplo, sobre o total de indivíduos incluídos em cada grupo, de participantes que desenvolveram a condição de interesse ao longo do acompanhamento, bem como os critérios diagnósticos empregados na identificação dos casos. Este último ponto é agravado pelo fato de que as condições bucais avaliadas foram diagnosticadas com instrumentos variados, e não diretamente comparáveis entre si. O fato de os estudos revisados avaliarem desfechos distintos de saúde bucal também impossibilita a realização da metanálise.

\section{RESULTADOS}

Das 661 publicações identificadas, 601 foram excluídas, após a leitura dos títulos e dos resumos, por não preencherem os critérios de inclusão. Posteriormente à leitura na íntegra dos 60 estudos selecionados por intermédio dos resumos, 23 trabalhos foram excluídos pelos seguintes motivos: 3 foram publicados em outros idiomas (além do inglês, espanhol e português), 1 trabalho ${ }^{11}$ não foi localizado na íntegra, 14 foram excluídos por não atenderem aos critérios de inclusão, ou seja, não isolaram o efeito da intervenção educativa sobre o desfecho, comparando-a com sua ausência, ou não analisaram desfechos finais clínicos, como cárie e sangramento gengival, e 5 não possuíam dados necessários para a análise. Os autores dos cinco últimos estudos foram contatados via correio eletrônico com objetivo de obter as informações necessárias, porém não retornaram as mensagens. Dessa forma, 35 publicações ${ }^{12-45}$ foram efetivamente revisadas no 
presente estudo. No entanto, duas pesquisas possuíam mais de um par comparativo, ou seja, mais de um grupo experimental comparado a um grupo controle, o que permitiu a investigação separada dos dados. Sendo assim, 37 resultados constituíram objeto da presente análise (Figura 1), que poderiam gerar uma ou mais comparações entre pares de grupos sob análise.

Com relação ao local de publicação, países da Europa e os Estados Unidos lideraram com 11 (29,2\%) artigos cada. Foi também constatado que essas publicações se concentraram nos últimos anos analisados, especificamente entre 2009 e 2013. Do total, 35 (94,6\%) intervenções abordaram indivíduos de ambos os sexos, das quais $20(54,1 \%)$ restringiram a idade até os 10 anos. A duração das intervenções apresentou distribuição assimétrica (não normal, com p $<0,001$, de acordo com o teste de Shapiro-Wilk), tendo mediana de 6 meses e intervalo interquartil de 22 meses, considerando-se simultaneamente os grupos de intervenção e de controle. Vinte e cinco $(67,6 \%)$ estudos apresentaram grupo controle, dos quais 8 (32,0\%) também

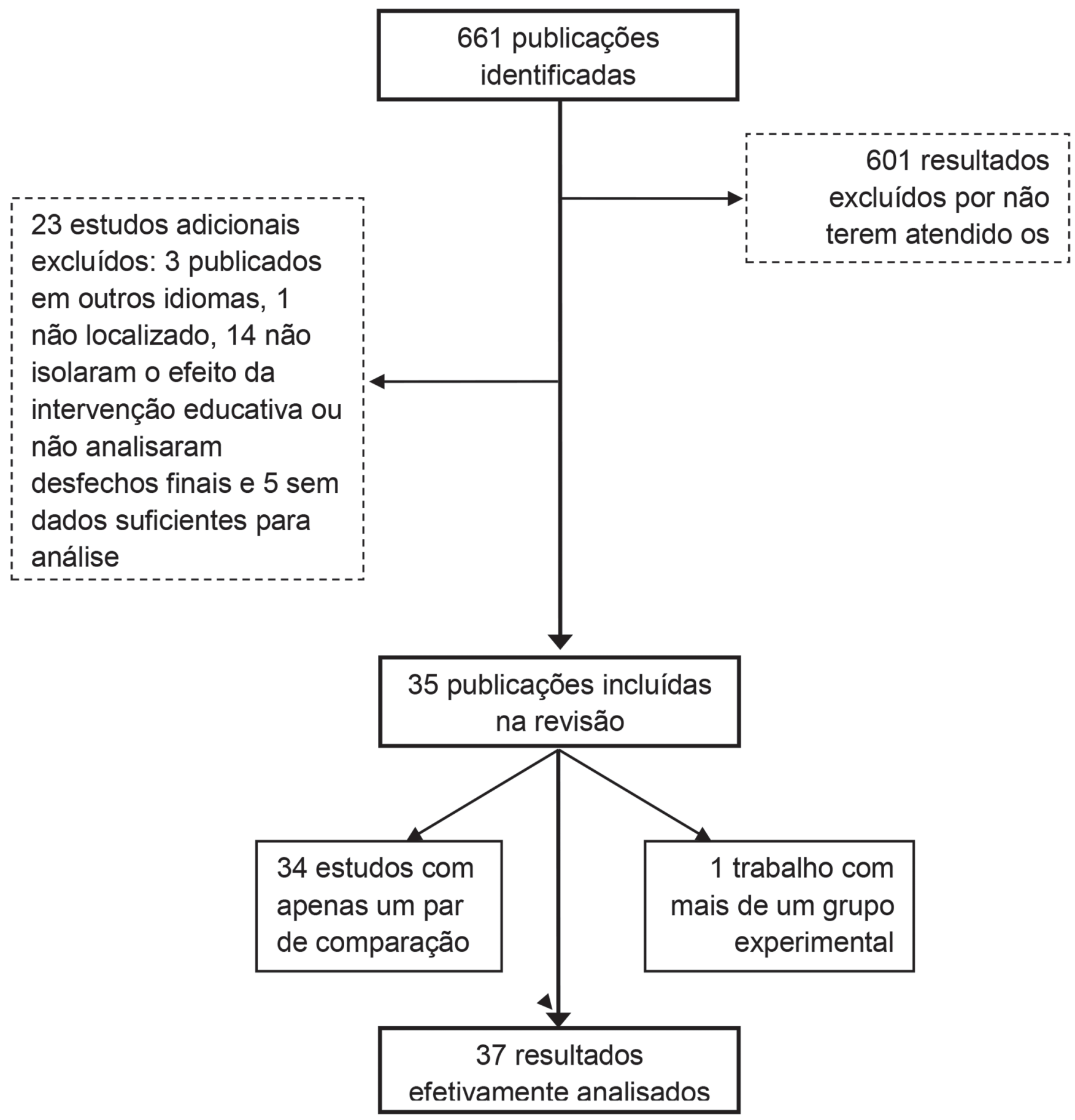

Figura 1. Fluxograma da revisão de literatura 
receberam intervenção sob a forma de instruções de higiene bucal; $31(83,8 \%)$ estudos não realizaram qualquer acompanhamento clínico dos participantes durante a intervenção, enquanto apenas $4(10,8 \%)$ artigos avaliaram os efeitos da intervenção em longo prazo (após seis meses do término da investigação). Dos $25(67,6 \%)$ artigos que informaram o profissional que realizou a intervenção, 15 (60,0\%) tiveram como referência o cirurgião-dentista. Com relação ao material educativo, os mais frequentemente empregados foram os folhetos e os fôlderes (13 estudos, 35,1\%), seguidos por recursos audiovisuais e a escovação supervisionada, ambos utilizados em 6 estudos cada (16,2\% para cada recurso) (Tabela 1$)$.

O desfecho mais investigado foi placa, avaliado em 25 (67,6\%) estudos, seguido por sangramento gengival (16 estudos, 43,2\%), cárie (10 estudos, 27,0\%), cálculo dentário e profundidade à sondagem (1 estudo cada). A maioria dos desfechos analisados apresentou um resultado melhor para o grupo que sofreu a intervenção educativa, quando comparado ao grupo controle. O sangramento gengival e a cárie apresentaram os maiores percentuais de redução ou menor incremento, respectivamente (Tabela 2). Nos 37 estudos revisados, observou-se que as intervenções produziram resultados clínicos positivos mas inconsistentes em curto prazo, incluindo redução de placa bacteriana (melhor resultado [MR] $-88,9 \%$; pior resultado $[P R]+29,7 \%$ ), sangramento gengival (MR $-77,8 \%$; PR $+72,2 \%$ ) e menor incremento de cárie (MR -39,5\%; PR +961,1\%) e de cálculo dentário (+16,2\%). A placa apresentou redução maior para os grupos que sofreram intervenção, comparado aos grupos controle; a cárie teve menor incremento no grupo intervenção, assim como o cálculo. O sangramento gengival também teve maior redução nos grupos que receberam intervenção, assim como a profundidade à sondagem. A avaliação de placa na mucosa demonstrou que o grupo controle apresentou resultado mais favorável em relação àquele que recebeu a intervenção educativa, com uma redução de 32,1 pontos percentuais maior que seu par (-48,4\% para o grupo controle e - 16,3 para grupo intervenção) (Tabela 2). Entretanto, essa avaliação foi restrita a apenas um estudo. Resultados similares foram encontrados ao considerarmos o conjunto das avaliações clínicas, ou seja, no lugar de considerar apenas o resultado referente à primeira e à última avaliação bucal, todos os dados clínicos disponíveis ao longo da intervenção foram incluídos na análise, como pode ser observado na Tabela 2.

Entre os participantes que receberam intervenção educativa e acompanhamento clínico intensivo, ou seja, os acompanhados durante o período da intervenção que pertenciam ao grupo intervenção, observou-se maior redução (não estatisticamente significativa, segundo os dados dos próprios estudos originais) de todos os agravos avaliados. Contudo, apenas seis artigos realizaram um acompanhamento clínico dos participantes ao
Tabela 1. Características das intervenções educativas empregadas nos estudos analisados

\begin{tabular}{|c|c|c|}
\hline & $\mathbf{N}$ & $\%$ \\
\hline \multicolumn{3}{|l|}{ Tempo de duração em meses } \\
\hline $0-2$ & 9 & 25,7 \\
\hline $3-5$ & 8 & 22,9 \\
\hline $6-10$ & 3 & 8,6 \\
\hline $11-20$ & 4 & 11,4 \\
\hline $21-30$ & 6 & 17,1 \\
\hline 31 ou mais & 5 & 14,3 \\
\hline \multicolumn{3}{|l|}{ Presença de grupo controle } \\
\hline $\operatorname{Sim}$ & 25 & 67,6 \\
\hline Não & 12 & 32,4 \\
\hline \multicolumn{3}{|c|}{$\begin{array}{l}\text { Quantidade de grupos analisados (incluindo } \\
\text { grupo controle) }\end{array}$} \\
\hline Um & 6 & 16,2 \\
\hline Dois & 22 & 59,5 \\
\hline Três & 6 & 16,2 \\
\hline Quatro & 3 & 8,1 \\
\hline \multicolumn{3}{|l|}{ Acompanhamento clínico* } \\
\hline Sim & 6 & 16,2 \\
\hline Não & 31 & 83,8 \\
\hline \multicolumn{3}{|l|}{ Avaliação em longo prazo** } \\
\hline Sim & 4 & 10,8 \\
\hline Não & 33 & 89,2 \\
\hline \multicolumn{3}{|c|}{ Profissional que realizou a intervenção } \\
\hline Dentista & 15 & 40,5 \\
\hline Higienista & 10 & 27,0 \\
\hline Professor & 3 & 8,1 \\
\hline Agente comunitário & 2 & 5,4 \\
\hline Enfermeiro & 1 & 2,7 \\
\hline Outro & 3 & 8,1 \\
\hline Não informado & 12 & 32,4 \\
\hline \multicolumn{3}{|c|}{$\begin{array}{l}\text { Material que foi utilizado no grupo de } \\
\text { intervenção*** }\end{array}$} \\
\hline Folheto & 13 & 35,1 \\
\hline Escovação supervisionada & 6 & 16,2 \\
\hline Audiovisual & 6 & 16,2 \\
\hline Pôsteres & 5 & 13,5 \\
\hline Telefone & 5 & 13,5 \\
\hline Jogos & 2 & 5,4 \\
\hline Mensagem de celular & 1 & 2,7 \\
\hline Não informado & 11 & 29,2 \\
\hline Outro & 9 & 24,4 \\
\hline Total & 37 & 100,0 \\
\hline
\end{tabular}

${ }^{*}$ Houve mais de uma coleta de dados durante a intervenção sem intervenção clínica; ${ }^{* *}$ Após, no mínimo, seis meses do término da intervenção; ${ }^{* *}$ Alguns grupos utilizaram mais de um material citado acima

longo do estudo. Quando os participantes do grupo controle foram acompanhados clinicamente, observou-se que houve redução da ocorrência de sangramento gengival. Os estudos avaliando placa acumulada na mucosa, cálculo e sangramento gengival não empregaram acompanhamento clínico. 
Tabela 2. Características dos estudos avaliando intervenções educativas sobre desfechos de saúde bucal incluídos na revisão

\begin{tabular}{|c|c|c|c|c|c|c|}
\hline $\begin{array}{c}\text { Ano de } \\
\text { publicação }\end{array}$ & $\begin{array}{l}\text { Sobrenome do } \\
\text { primeiro autor }\end{array}$ & $\begin{array}{c}\text { Tempo de } \\
\text { duração da } \\
\text { intervenção } \\
\text { (meses) }\end{array}$ & $\begin{array}{c}\text { Sexo dos } \\
\text { participantes }\end{array}$ & $\begin{array}{l}\text { Desfecho } \\
\text { avaliado }\end{array}$ & $\begin{array}{c}\text { Impacto relativo } \\
\text { da intervenção } \\
\text { sobre o agravo } \\
(\%) *\end{array}$ & $\begin{array}{c}\text { Impacto relativo } \\
\text { da intervenção } \\
\text { sobre o agravo } \\
(\%)^{* *}\end{array}$ \\
\hline 2013 & Mbawalla & 23 & $\begin{array}{l}\text { Masculino e } \\
\text { feminino }\end{array}$ & Cálculo & 16,2 & 16,2 \\
\hline 2005 & Al-Jundi & 48 & $\begin{array}{l}\text { Masculino e } \\
\text { feminino }\end{array}$ & Cárie & 0,4 & 0,4 \\
\hline 2005 & Al-Jundi & 48 & $\begin{array}{l}\text { Masculino e } \\
\text { feminino }\end{array}$ & Cárie & 0,6 & 0,6 \\
\hline 2003 & Blinkhorn & 24 & $\begin{array}{l}\text { Masculino e } \\
\text { feminino }\end{array}$ & Cárie & 63,5 & 63,5 \\
\hline 2004 & Peng & 24 & $\begin{array}{l}\text { Masculino e } \\
\text { feminino }\end{array}$ & Cárie & $-17,9$ & 48,7 \\
\hline 2004 & Petersen & 36 & $\begin{array}{l}\text { Masculino e } \\
\text { feminino }\end{array}$ & Cárie & $-39,5$ & $-39,5$ \\
\hline 2003 & Vachirarojpisan & 12 & $\begin{array}{l}\text { Masculino e } \\
\text { feminino }\end{array}$ & Cárie & 961,1 & 961,1 \\
\hline 2003 & Almomani & 1 & $\begin{array}{l}\text { Masculino e } \\
\text { feminino }\end{array}$ & Placa & $-26,7$ & $-26,7$ \\
\hline 2009 & Almomani & 2 & $\begin{array}{l}\text { Masculino e } \\
\text { feminino }\end{array}$ & Placa & $-35,7$ & $-32,2$ \\
\hline 2007 & Antonio & 30 & $\begin{array}{l}\text { Masculino e } \\
\text { feminino }\end{array}$ & Placa & $-5,1$ & $-12,6$ \\
\hline 2011 & Anttonen & 12 & $\begin{array}{l}\text { Masculino e } \\
\text { feminino }\end{array}$ & Placa & $-0,4$ & $-0,4$ \\
\hline 2003 & Biesbrock & 1 & $\begin{array}{l}\text { Masculino e } \\
\text { feminino }\end{array}$ & Placa & $-29,5$ & $-29,5$ \\
\hline 2005 & Biesbrock & 1 & $\begin{array}{l}\text { Masculino e } \\
\text { feminino }\end{array}$ & Placa & 29,7 & 29,7 \\
\hline 2008 & Bizarra & 3 & $\begin{array}{l}\text { Masculino e } \\
\text { feminino }\end{array}$ & Placa & $-34,3$ & $-34,3$ \\
\hline 2009 & Clarkson & 2 & $\begin{array}{l}\text { Masculino e } \\
\text { feminino }\end{array}$ & Placa & $-47,4$ & $-47,4$ \\
\hline 2009 & Clarkson & 2 & $\begin{array}{l}\text { Masculino e } \\
\text { feminino }\end{array}$ & Placa & $-33,5$ & $-33,5$ \\
\hline 2011 & Esfahanizadeh & 6 & $\begin{array}{l}\text { Masculino e } \\
\text { feminino }\end{array}$ & Placa & $-13,3$ & $-13,3$ \\
\hline 2012 & Hebbal & 19 & $\begin{array}{l}\text { Masculino e } \\
\text { feminino }\end{array}$ & Placa & $-55,3$ & $-55,3$ \\
\hline 2007 & Hugoson & Dado indisponível & $\begin{array}{l}\text { Masculino e } \\
\text { feminino }\end{array}$ & Placa & $-68,4$ & $-61,9$ \\
\hline 2005 & Jonsson & 3 & $\begin{array}{l}\text { Masculino e } \\
\text { feminino }\end{array}$ & Placa & $-57,6$ & $-57,6$ \\
\hline 2013 & Lam & 0 & $\begin{array}{l}\text { Masculino e } \\
\text { feminino }\end{array}$ & Placa & $-40,0$ & $-40,0$ \\
\hline 2013 & Marinho & 4 & $\begin{array}{l}\text { Masculino e } \\
\text { feminino }\end{array}$ & Placa & 25,9 & 25,9 \\
\hline 2013 & Mbawalla & 23 & $\begin{array}{l}\text { Masculino e } \\
\text { feminino }\end{array}$ & Placa & $-39,3$ & $-39,3$ \\
\hline 2007 & Ramseier & 1 & $\begin{array}{l}\text { Masculino e } \\
\text { feminino }\end{array}$ & Placa & $-8,1$ & $-8,1$ \\
\hline 2009 & Rodrigues & 3 & $\begin{array}{l}\text { Masculino e } \\
\text { feminino }\end{array}$ & Placa & $-32,2$ & $-30,1$ \\
\hline 2009 & Saied-Moallemi & 3 & $\begin{array}{l}\text { Masculino e } \\
\text { feminino }\end{array}$ & Placa & $-88,9$ & $-88,9$ \\
\hline
\end{tabular}

${ }^{*}$ Impacto da intervenção, levando-se em conta somente os registros clínicos inicial e final dos participantes; ${ }^{* *}$ Impacto da intervenção, incluindo-se todas as informações clínicas disponíveis dos indivíduos investigados compreendendo aquelas intermediárias ao início e ao término da intervenção, além da inicial e da final 
Tabela 2. Continuação...

\begin{tabular}{|c|c|c|c|c|c|c|}
\hline $\begin{array}{c}\text { Ano de } \\
\text { publicação }\end{array}$ & $\begin{array}{l}\text { Sobrenome do } \\
\text { primeiro autor }\end{array}$ & $\begin{array}{c}\text { Tempo de } \\
\text { duração da } \\
\text { intervenção } \\
\text { (meses) }\end{array}$ & $\begin{array}{c}\text { Sexo dos } \\
\text { participantes }\end{array}$ & $\begin{array}{l}\text { Desfecho } \\
\text { avaliado }\end{array}$ & $\begin{array}{c}\text { Impacto relativo } \\
\text { da intervenção } \\
\text { sobre o agravo } \\
(\%) *\end{array}$ & $\begin{array}{c}\text { Impacto relativo } \\
\text { da intervenção } \\
\text { sobre o agravo } \\
(\%) * *\end{array}$ \\
\hline 2011 & Sharma & 4 & $\begin{array}{l}\text { Masculino e } \\
\text { feminino }\end{array}$ & Placa & $-23,6$ & $-23,6$ \\
\hline 2007 & Slomkowska & 1 & $\begin{array}{l}\text { Masculino e } \\
\text { feminino }\end{array}$ & Placa & $-55,1$ & $-55,1$ \\
\hline 2006 & Yalcinkaya & 9 & $\begin{array}{l}\text { Masculino e } \\
\text { feminino }\end{array}$ & Placa & $-34,6$ & $-25,6$ \\
\hline 2009 & Yazdani & 26 & $\begin{array}{l}\text { Masculino e } \\
\text { feminino }\end{array}$ & Placa & $-37,7$ & $-37,7$ \\
\hline 2009 & Ziebolz & 3 & Masculino & Placa & $-48,5$ & $-48,5$ \\
\hline 2009 & Saied-Moallemi & 3 & $\begin{array}{l}\text { Masculino e } \\
\text { feminino }\end{array}$ & Placa na mucosa & $-16,3$ & $-16,3$ \\
\hline 2005 & Jonsson & 3 & $\begin{array}{l}\text { Masculino e } \\
\text { feminino }\end{array}$ & $\begin{array}{c}\text { Profundidade à } \\
\text { sondagem }\end{array}$ & $-60,0$ & $-60,0$ \\
\hline 2007 & Antonio & 30 & $\begin{array}{l}\text { Masculino e } \\
\text { feminino }\end{array}$ & $\begin{array}{c}\text { Sangramento } \\
\text { gengival }\end{array}$ & $-77,8$ & $-77,8$ \\
\hline 2003 & Biesbrock & 1 & $\begin{array}{l}\text { Masculino e } \\
\text { feminino }\end{array}$ & $\begin{array}{c}\text { Sangramento } \\
\text { gengival }\end{array}$ & $-51,3$ & $-51,3$ \\
\hline 2005 & Biesbrock & 1 & $\begin{array}{l}\text { Masculino e } \\
\text { feminino }\end{array}$ & $\begin{array}{c}\text { Sangramento } \\
\text { gengival }\end{array}$ & $-20,2$ & $-20,2$ \\
\hline 2009 & Clarkson & 2 & $\begin{array}{l}\text { Masculino e } \\
\text { feminino }\end{array}$ & $\begin{array}{c}\text { Sangramento } \\
\text { gengival }\end{array}$ & $-44,0$ & $-44,0$ \\
\hline 2009 & Clarkson & 2 & $\begin{array}{l}\text { Masculino e } \\
\text { feminino }\end{array}$ & $\begin{array}{l}\text { Sangramento } \\
\text { gengival }\end{array}$ & $-46,5$ & $-46,5$ \\
\hline 2011 & Esfahanizadeh & 6 & $\begin{array}{l}\text { Masculino e } \\
\text { feminino }\end{array}$ & $\begin{array}{l}\text { Sangramento } \\
\text { gengival }\end{array}$ & $-10,8$ & $-10,8$ \\
\hline 2007 & Hugoson & Dado indisponível & $\begin{array}{l}\text { Masculino e } \\
\text { feminino }\end{array}$ & $\begin{array}{c}\text { Sangramento } \\
\text { gengival }\end{array}$ & $-52,3$ & $-47,9$ \\
\hline 2005 & Jonsson & 3 & $\begin{array}{l}\text { Masculino e } \\
\text { feminino }\end{array}$ & $\begin{array}{c}\text { Sangramento } \\
\text { gengival }\end{array}$ & $-47,9$ & $-47,9$ \\
\hline 2013 & Lam & 0 & $\begin{array}{l}\text { Masculino e } \\
\text { feminino }\end{array}$ & $\begin{array}{c}\text { Sangramento } \\
\text { gengival }\end{array}$ & 6,0 & 6,0 \\
\hline 2013 & Marinho & 4 & $\begin{array}{l}\text { Masculino e } \\
\text { feminino }\end{array}$ & $\begin{array}{c}\text { Sangramento } \\
\text { gengival }\end{array}$ & $-75,0$ & $-75,0$ \\
\hline 2013 & Mbawalla & 23 & $\begin{array}{l}\text { Masculino e } \\
\text { feminino }\end{array}$ & $\begin{array}{c}\text { Sangramento } \\
\text { gengival }\end{array}$ & $-25,0$ & $-25,0$ \\
\hline 2012 & Nammontri & 3 & $\begin{array}{l}\text { Masculino e } \\
\text { feminino }\end{array}$ & $\begin{array}{c}\text { Sangramento } \\
\text { gengival }\end{array}$ & 72,2 & 72,2 \\
\hline 2004 & Petersen & 36 & $\begin{array}{l}\text { Masculino e } \\
\text { feminino }\end{array}$ & $\begin{array}{c}\text { Sangramento } \\
\text { gengival }\end{array}$ & 61,2 & 61,2 \\
\hline 2009 & Samson & 72 & $\begin{array}{l}\text { Masculino e } \\
\text { feminino }\end{array}$ & $\begin{array}{c}\text { Sangramento } \\
\text { gengival }\end{array}$ & $-27,8$ & $-27,8$ \\
\hline 2006 & Yalcinkaya & 9 & $\begin{array}{l}\text { Masculino e } \\
\text { feminino }\end{array}$ & $\begin{array}{c}\text { Sangramento } \\
\text { gengival }\end{array}$ & $-31,6$ & $-25,1$ \\
\hline 2009 & Yazdani & 26 & $\begin{array}{l}\text { Masculino e } \\
\text { feminino }\end{array}$ & $\begin{array}{l}\text { Sangramento } \\
\text { gengival }\end{array}$ & $-56,1$ & $-56,1$ \\
\hline
\end{tabular}

${ }^{*}$ Impacto da intervenção, levando-se em conta somente os registros clínicos inicial e final dos participantes; ${ }^{* *}$ Impacto da intervenção, incluindo-se todas as informações clínicas disponíveis dos indivíduos investigados compreendendo aquelas intermediárias ao início e ao término da intervenção, além da inicial e da final 


\section{DISCUSSÃO}

Dos 35 artigos analisados, uma expressiva parte se concentrou nos Estados Unidos e em países desenvolvidos da Europa, a maioria não apresentou qualquer acompanhamento clínico durante a intervenção e apenas 25 possuíam grupo controle, dos quais 8 também receberam intervenção sob a forma de instruções de higiene bucal. Somente quatro trabalhos avaliaram o possível efeito da intervenção sobre os desfechos de saúde bucal em longo prazo, ou seja, após seis meses do término do estudo. O cirurgião-dentista foi o profissional de escolha para aplicação da intervenção na maioria dos estudos, e os materiais mais comumente empregados foram os folhetos e os fôlderes.

As intervenções resultaram em uma melhora para a maioria das condições avaliadas, quando comparadas ao grupo controle, e obtiveram resultados inconsistentes em curto prazo, como redução de placa bacteriana, sangramento gengival, menor incremento de cárie dentária e de cálculo. Porém o sangramento à sondagem apresentou pouca diferença entre os grupos, enquanto o desfecho placa acumulada na mucosa apresentou um resultado melhor para o grupo controle. Quando comparados os resultados iniciais, baseados apenas nos dados da primeira e da última avaliação bucal, com os resultados da segunda análise, que utilizam o conjunto de todas as avaliações clínicas realizadas nos participantes, constataram-se efeitos similares. Estudos cujos participantes foram submetidos a avaliações clínicas periódicas para coleta dos resultados apresentaram melhoras mais expressivas. Entretanto, ao analisar exclusivamente os indivíduos dos grupos controle, apenas o índice de sangramento gengival mostrou resultado positivo, quando os participantes receberam acompanhamento clínico intensivo.

As intervenções educativas para prevenção, controle ou redução de agravos bucais não apresentaram efeitos consistentes do ponto de vista clínico, quando comparadas a outras intervenções, por exemplo, a fluoretação das águas de abastecimento, que foi responsável pela redução de 50,0 a 60,0\% da cárie dentária em crianças ${ }^{46}$. Estratégias de abordagens universais, como é o caso da fluoretação das águas, traduzem-se em resultados mais promissores em relação à prevenção ou ao controle de agravos bucais, pois atingem uma grande parcela da população, promovendo mudanças significativas na incidência desses agravos, quando comparadas àquelas voltadas a grupos e indivíduos de alto risco ${ }^{47}$. Contudo, essas estratégias pressupõem o acesso universal da população a determinada intervenção, o que pode não ocorrer. Ainda assim, evidências apontam para existência de desigualdades em relação à distribuição da cárie dentária, por exemplo, mesmo em áreas sem fluoretação das águas ${ }^{48,49}$, o que indica que intervenções educativas e preventivas focalizadas em grupos mais acometidos podem ser eventualmente necessárias para o enfrentamento dos problemas bucais.
Durante a busca pelos artigos que atendessem aos critérios de inclusão, observou-se que, embora houvesse um número considerável de artigos sobre o tema, grande parte deles limitava-se a avaliar apenas os desfechos intermediários, como o acúmulo de conhecimento pelos participantes, impossibilitando a análise do impacto da intervenção nos desfechos finais (clínicos). Além disso, os artigos revisados apresentaram problemas metodológicos importantes. Entre os principais problemas destacados, estão a falta de grupo controle (ausente em 10 estudos), a inexistência de padronização da coleta de dados e a ampla variação do método de avaliação do desfecho (os estudos utilizavam índices diferentes para mensurar o mesmo desfecho), que dificultam a análise e colocam em dúvida a qualidade da evidência produzida a partir dos estudos analisados. Dos 18 estudos que avaliaram placa, por exemplo, 8 não informaram qual o método de avaliação usado, e os outros 10 que o fizeram, empregaram 5 critérios distintos de avaliação. Nesse sentido, os problemas mais comuns das intervenções de saúde bucal se relacionam à qualidade, à validade e à comparabilidade das avaliações.

Outra limitação dos estudos analisados é falta de informações sobre as intervenções realizadas, o que fez com que algumas das análises não incluíssem todos os artigos selecionados na revisão. Além disso, a escassez de estudos avaliando o resultado nos desfechos em longo prazo não nos permitiu afirmar se as melhoras poderiam ser sustentáveis com o passar do tempo. Tendo em vista que, em curto prazo, não houve efeitos detectáveis das intervenções sobre os desfechos avaliados, provavelmente o mesmo seria observado caso os estudos tivessem empregado períodos maiores de acompanhamento e de observação. Dos 35 estudos abordados, apenas 4 avaliaram resultados em longo prazo; 2, após seis meses do término da intervenção; 1 , após 12 meses; e 1, após 69 meses. Apenas 1 estudo observou a permanência dos resultados positivos da intervenção (embora sem significância estatística, segundo dados do próprio artigo original), enquanto os demais demonstraram sucesso parcial em longo prazo.

Vale ressaltar que, ainda que não tenham sido foco exclusivo desta pesquisa, os estudos baseados na teoria KAP (knowledge, attitude and practice) partem do princípio de que a detenção do conhecimento é suficiente para uma mudança de atitude e, finalmente, de comportamentos relacionados à saúde. Essa visão desconsidera diversos fatores sociais, culturais e econômicos que contribuem para o desenvolvimento, manutenção e mudança de comportamento, reduzindo-se a uma visão culpabilizadora e simplista dos processos relacionados ao padrão de comportamentos e aos determinantes sociais do processo saúde-doença. Por sua vez, o modelo ecológico social de promoção da saúde propõe que a criação de um ambiente propício para a mudança é fundamental para facilitar a adoção de comportamentos saudáveis. Sendo assim, as intervenções 
não devem visar apenas os indivíduos, mas afetar aspectos que influenciem o comportamento em saúde, relações interpessoais, fatores organizacionais e ambientais ${ }^{50}$. As relações entre conhecimento, consciência da necessidade de mudar, intenção para mudar e uma efetiva mudança no comportamento são muito complexas, por isso, o comportamento em saúde sustentando mudança envolve diversas ações e adaptações ao longo do tempo. A realização de mudanças iniciais de comportamento e a manutenção delas requerem diferentes tipos de estratégias, que devem ser cuidadosamente estudadas e planejadas ${ }^{50}$.

Há uma necessidade de mais pesquisas com estudos de base populacional e bem delineados sobre o resultado imediato e em longo prazo, assim como uma avaliação detalhada do processo de implementação da intervenção, preferencialmente, por meio de abordagens de avaliação pluralistas (quantitativa e/ou qualitativa $)^{51}$. Também é necessário que os estudos futuros empreguem modelos teóricos robustos, descrição e planejamento detalhado das intervenções ${ }^{52}$.

Entre as limitações desta revisão, destaca-se a língua de publicação dos estudos revisados - três pesquisas não puderam ser avaliadas por estarem disponíveis em línguas inacessíveis aos autores. A estratégia de análise do impacto das intervenções educativas foi igualmente limitada, visto que não foi levada em conta a heterogeneidade dos estudos, tanto dos desfechos quanto das abordagens. A busca eletrônica também não foi exaustiva, restringindo-se apenas à base de dados MEDLINE via PubMed, além de não ter sido realizada procura manual por artigos. Da mesma forma, os estudos que não puderam ser encontrados ou não tiveram seus resultados disponibilizados, após contato com os autores, também não foram incluídos nas análises, o que representa uma limitação adicional do trabalho de revisão.

Em síntese, as intervenções educativas analisadas nesta revisão não se preocuparam em incorporar uma visão ampliada do modelo de promoção da saúde, e a efetividade delas na prevenção ou na redução de diferentes agravos bucais não pôde ser claramente demonstrada. O presente estudo buscou revisar de forma ampla todas as formas de intervenções educativas em saúde bucal, analisando seu impacto em cada publicação. Espera-se que os resultados e as recomendações aqui destacadas resultem em intervenções de melhor qualidade e, no limite, uma saúde bucal melhor para a população por meio de atividades de promoção de saúde mais efetivas.

\section{REFERÊNCIAS}

1. Petersen PE, Bourgeois D, Ogawa H, Estupinan-Day S, Ndiaye C. The global burden of oral diseases and risks to oral health. Bull World Health Organ. 2005;83(9):661-9. PMid:16211157.

2. Brasil. Ministério da Saúde. SB Brasil 2010:0 Pequisa Nacional de Saúde Bucal: resultados principais. Brasília: Secretaria de Atenção à Saúde/ Secretaria de Vigilância em Saúde; 2012.

3. Andrade FB, Lebrao ML, Santos JL, Duarte YA. Correlates of change in self-perceived oral health among older adults in Brazil: findings from the health, well-being and aging study. J Am Dent Assoc. 2012;143(5):488-95. http://dx.doi.org/10.14219/jada.archive.2012.0209. PMid:22547720.

4. Peres KG, Cascaes AM, Leao AT, Cortes MI, Vettore MV. Sociodemographic and clinical aspects of quality of life related to oral health in adolescents. Rev Saude Publica. 2013;47(Supl 3):19-28. http://dx.doi.org/10.1590/ S0034-8910.2013047004361. PMid:24626578.

5. Marinho VC, Higgins JP, Logan S, Sheiham A. Topical fluoride (toothpastes, mouthrinses, gels or varnishes) for preventing dental caries in children and adolescents. Cochrane Database Syst Rev. 2003;(4):CD002782. PMid:14583954

6. Marinho VC, Higgins JP, Sheiham A, Logan S. Combinations of topical fluoride (toothpastes, mouthrinses, gels, varnishes) versus single topical fluoride for preventing dental caries in children and adolescents. Cochrane Database Syst Rev. 2004;(1):CD002781. PMid:14973992.

7. Sheiham A, James WP. A new understanding of the relationship between sugars, dental caries and fluoride use: implications for limits on sugars consumption. Public Health Nutr. 2014;17(10):2176-84. http://dx.doi. org/10.1017/S136898001400113X. PMid:24892213.
8. Kay EJ, Locker D. Is dental health education effective? A systematic review of current evidence. Community Dent Oral Epidemiol. 1996;24(4):231-5. http://dx.doi.org/10.1111/j.1600-0528.1996.tb00850.x. PMid:8871028.

9. Satur JG, Gussy MG, Morgan MV, Calache H, Wright C. Review of the evidence for oral health promotion effectiveness. Health Educ J. 2010;69(3):257-66. http://dx.doi.org/10.1177/0017896909349240.

10. Watt RG, Marinho VC. Does oral health promotion improve oral hygiene and gingival health? Periodontol 2000. 2005;37(1):35-47. http://dx.doi. org/10.1111/j.1600-0757.2004.03796.x. PMid:15655024.

11. Almas K, Al-Lazzam S, Al-Quadairi A. The effect of oral hygiene instructions on diabetic type 2 male patients with periodontal diseases. J Contemp Dent Pract. 2003;4(3):24-35. PMid:12937594.

12. Al-Jundi SH, Hammad M, Alwaeli H. The efficacy of a school-based caries preventive program: a 4-year study. Int J Dent Hyg. 2006;4(1):30-4. http:// dx.doi.org/10.1111/j.1601-5037.2006.00156.x. PMid:16451437.

13. Almomani F, Brown C, Williams KB. The effect of an oral health promotion program for people with psychiatric disabilities. Psychiatr Rehabil J. 2006;29(4):274-81. http://dx.doi.org/10.2975/29.2006.274.281. PMid:16689038.

14. Almomani F, Williams K, Catley D, Brown C. Effects of an oral health promotion program in people with mental illness. J Dent Res. 2009;88(7):64852. http://dx.doi.org/10.1177/0022034509338156. PMid:19605879.

15. Antonio AG, Kelly A, Valle DD, Vianna RB, Quintanilha LE. Long-term effect of an oral health promotion program for schoolchildren after the interruption of educational activities. J Clin Pediatr Dent. 2007;32(1):37-41. http://dx.doi.org/10.17796/jcpd.32.1.q35505m1u07244p1. PMid:18274468. 
16. Anttonen V, Seppa L, Niinimaa A, Hausen H. Dietary and oral hygiene intervention in secondary school pupils. Int J Paediatr Dent. 2011;21(2):818. http://dx.doi.org/10.1111/j.1365-263X.2010.01095.x. PMid:20731735.

17. Biesbrock AR, Walters PA, Bartizek RD. Initial impact of a national dental education program on the oral health and dental knowledge of children. J Contemp Dent Pract. 2003;4(2):1-10. PMid:12761585.

18. Biesbrock AR, Walters PA, Bartizek RD. Short-term impact of a national dental education program on children's oral health and knowledge. J Clin Dent. 2004;15(4):93-7. PMid:15794453.

19. Bizarra F, Ribeiro S. Improving toothbrushing behaviour in an institution for the disabled in Lisbon, Portugal. Int J Dent Hyg. 2009;7(3):182-7. http:// dx.doi.org/10.1111/j.1601-5037.2009.00387.x. PMid:19659714.

20. Blinkhorn AS, Gratrix D, Holloway PJ, Wainwright-Stringer YM, Ward SJ, Worthington HV. A cluster randomised, controlled trial of the value of dental health educators in general dental practice. Br Dent J. 2003;195(7):395-400. http://dx.doi.org/10.1038/sj.bdj.4810566. PMid:14551632.

21. Clarkson JE, Young L, Ramsay CR, Bonner BC, Bonetti D. How to influence patient oral hygiene behavior effectively. J Dent Res. 2009;88(10):933-7. http://dx.doi.org/10.1177/0022034509345627. PMid:19783802.

22. Davies GM, Duxbury JT, Boothman NJ, Davies RM. Challenges associated with the evaluation of a dental health promotion programme in a deprived urban area. Community Dent Health. 2007;24(2):117-21. PMid:17615828.

23. Esfahanizadeh N. Dental health education programme for 6-year-olds: a cluster randomised controlled trial. Eur J Paediatr Dent. 2011;12(3):167-70. PMid:22077684.

24. Hebbal M, Ankola AV. Development of a new technique (ATP) for training visually impaired children in oral hygiene maintenance. Eur Arch Paediatr Dent. 2012;13(5):244-7. http://dx.doi.org/10.1007/BF03262878. PMid:23043880.

25. Hugoson A, Lundgren D, Asklow B, Borgklint G. Effect of three different dental health preventive programmes on young adult individuals: a randomized, blinded, parallel group, controlled evaluation of oral hygiene behaviour on plaque and gingivitis. J Clin Periodontol. 2007;34(5):407-15. http://dx.doi.org/10.1111/j.1600-051X.2007.001069.x. PMid:17448044.

26. Jönsson B, Lindberg P, Oscarson N, Ohrn K. Improved compliance and self-care in patients with periodontitis: a randomized control trial. Int J Dent Hyg. 2006;4(2):77-83. http://dx.doi.org/10.1111/j.1601-5037.2006.00175.x. PMid:16637909.

27. Lam OL, McMillan AS, Samaranayake LP, Li LS, McGrath C. Randomized clinical trial of oral health promotion interventions among patients following stroke. Arch Phys Med Rehabil. 2013;94(3):435-43. http://dx.doi. org/10.1016/j.apmr.2012.10.024. PMid:23127306.

28. Mariño R, Calache H, Morgan M. A community-based culturally competent oral health promotion for migrant older adults living in Melbourne, Australia. J Am Geriatr Soc. 2013;61(2):270-5. http://dx.doi.org/10.1111/ jgs.12078. PMid:23320643.

29. Martignon S, Gonzalez MC, Santamaria RM, Jacome-Lievano S, Munoz Y, Moreno P. Oral-health workshop targeted at 0-5-yr. old deprived children's parents and caregivers: effect on knowledge and practices. J Clin Pediatr Dent. 2006;31(2):104-8. http://dx.doi.org/10.17796/jcpd.31.2.a20j230572178wp5. PMid:17315804.

30. Mbawalla H, Masalu JR, Masatu M, Astrom AN. Changes in adolescents' oral health status following oral health promotion activities in Tanzania. Acta Odontol Scand. 2013;71(2):333-42. http://dx.doi.org/10.3109/00016

\subsubsection{PMid:22563991.}

31. Nammontri O, Robinson PG, Baker SR. Enhancing oral health via sense of coherence: a cluster-randomized trial. J Dent Res. 2013;92(1):26-31. http://dx.doi.org/10.1177/0022034512459757. PMid:23018820.

32. Peng B, Petersen PE, Bian Z, Tai B, Jiang H. Can school-based oral health education and a sugar-free chewing gum program improve oral health? Results from a two-year study in PR China. Acta Odontol Scand. 2004;62(6):32832. http://dx.doi.org/10.1080/00016350410010036. PMid:15848976.

33. Plutzer K, Spencer AJ. Efficacy of an oral health promotion intervention in the prevention of early childhood caries. Community Dent Oral Epidemiol. 2008;36(4):335-46. http://dx.doi.org/10.1111/j.1600-0528.2007.00414.x. PMid:19145720.

34. Ramseier CA, Leiggener I, Lang NP, Bagramian RA, Inglehart MR. Shortterm effects of hygiene education for preschool (kindergarten) children: a clinical study. Oral Health Prev Dent. 2007;5(1):19-24. PMid:17366757.

35. Rodrigues JA, Santos PA, Baseggio W, Corona SA, Palma-Dibb RG, Garcia PP. Oral hygiene indirect instruction and periodic reinforcements: effects on index plaque in schoolchildren. J Clin Pediatr Dent. 2009;34(1):31-4. http://dx.doi.org/10.17796/jcpd.34.1.n426k2862m42r67n. PMid:19953806.

36. Saied-Moallemi Z, Virtanen JI, Vehkalahti MM, Tehranchi A, Murtomaa H. School-based intervention to promote preadolescents' gingival health: a community trial. Community Dent Oral Epidemiol. 2009;37(6):518-26. http://dx.doi.org/10.1111/j.1600-0528.2009.00491.x. PMid:19694774.

37. Samson H, Berven L, Strand GV. Long-term effect of an oral healthcare programme on oral hygiene in a nursing home. Eur J Oral Sci. 2009;117(5):575 9. http://dx.doi.org/10.1111/j.1600-0722.2009.00673.x. PMid:19758255.

38. Sharma R, Hebbal M, Ankola AV, Murugabupathy V. Mobile-phone text messaging (SMS) for providing oral health education to mothers of preschool children in Belgaum City. J Telemed Telecare. 2011;17(8):432-6. http://dx.doi.org/10.1258/jtt.2011.110416. PMid:22025742.

39. Smiech-Slomkowska G, Jablonska-Zrobek J. The effect of oral health education on dental plaque development and the level of caries-related Streptococcus mutans and Lactobacillus spp. Eur J Orthod. 2007;29(2):15760. http://dx.doi.org/10.1093/ejo/cjm001. PMid:17488998.

40. Vachirarojpisan T, Shinada K, Kawaguchi Y. The process and outcome of a programme for preventing early childhood caries in Thailand. Community Dent Health. 2005;22(4):253-9. PMid:16379164.

41. Weinstein P, Harrison R, Benton T. Motivating parents to prevent caries in their young children: one-year findings. J Am Dent Assoc. 2004;135(6):7318. http://dx.doi.org/10.14219/jada.archive.2004.0299. PMid:15270155.

42. Whittle JG, Whitehead HF, Bishop CM. A randomised control trial of oral health education provided by a health visitor to parents of pre-school children. Community Dent Health. 2008;25(1):28-32. PMid:18435231.

43. Yalcinkaya SE, Atalay T. Improvement of oral health knowledge in a group of visually impaired students. Oral Health Prev Dent. 2006;4(4):243-53. PMid:17153646.

44. Yazdani R, Vehkalahti MM, Nouri M, Murtomaa H. School-based education to improve oral cleanliness and gingival health in adolescents in Tehran, Iran. Int J Paediatr Dent. 2009;19(4):274-81. http://dx.doi.org/10.1111/j.1365263X.2009.00972.x. PMid:19320914.

45. Ziebolz D, Herz A, Brunner E, Hornecker E, Mausberg RF. Individual versus group oral hygiene instruction for adults. Oral Health Prev Dent. 2009;7(1):93-9. PMid:19408821.

46. Centers for Disease Control and Prevention. Recommendations for using fluoride to prevent and control dental caries in the United States. Atlanta: CDC; 2001. p. 1-42. 
47. Batchelor PA, Sheiham A. The distribution of burden of dental caries in schoolchildren: a critique of the high-risk caries prevention strategy for populations. BMC Oral Health. 2006;6(3):3. http://dx.doi.org/10.1186/14726831-6-3. PMid:16448565.

48. Cardoso L, Rösing C, Kramer P, Costa CC, Costa Fo LC. Polarização da cárie em município sem água fluoretada. Cad Saude Publica. 2003;19(1):237-43. http://dx.doi.org/10.1590/S0102-311X2003000100026. PMid:12700803.

49. Margolis MQ, Hunt RJ, Vann Jr WF, Stewart PW. Distribution of primary tooth caries in first-grade children from two nonfluoridated US communities. Pediatr Dent. 1994;16(3):200-5. PMid:8058544.

50. Glanz K, Bishop DB. The role of behavioral science theory in development and implementation of public health interventions. Annu Rev Public
Health. 2010;31(1):399-418. http://dx.doi.org/10.1146/annurev. publhealth.012809.103604. PMid:20070207.

51. Petersen PE, Kwan S. Evaluation of community-based oral health promotion and oral disease prevention--WHO recommendations for improved evidence in public health practice. Community Dent Health. 2004;21(Supl):319-29. PMid:15617418.

52. Bartholomew LK, Mullen PD. Five roles for using theory and evidence in the design and testing of behavior change interventions. J Public Health Dent. 2011;71(Supl 1):S20-33. http://dx.doi.org/10.1111/j.1752-7325.2011.00223.x. PMid:21656946.

Recebido em: Set. 20, 2014

Aprovado em: Jul. 22, 2015 